\title{
Stent Coronário de Liga Cobalto-Cromo Concebido no Brasil: Achados Histológicos Preliminares em Modelo Experimental Porcino
}

\author{
Pedro A. Lemos', Francisco R. M. Laurindo', Spero P. Morato², Celso Takimura', \\ Carlos A. Campos' ${ }^{1}$, Paulo S. Gutierrez', Vera D. Aiello', Bruno Ctenas', Luciene M. dos Reis ${ }^{3}$, \\ Expedito E. Ribeiro', Eulógio E. Martinez'
}

\section{RESUMO}

Introdução: Este estudo objetiva avaliar em modelo experimental porcino um novo e pioneiro stent tubular de liga cobalto-cromo e de hastes finas, de concepção brasileira. Objetiva também relatar a experiência inicial de um centro de validação pré-clínica de dispositivos endovasculares instalado no País. Método: No total, 12 porcos domésticos foram submetidos a implante coronário de um novo stent de liga cobalto-cromo não-farmacológico (Scitech Produtos Médicos Ltda.). O stent possuía desenho em anéis corrugados com células curtas (aumento da homogeneidade da cobertura metálica), hastes finas (75 $\mu \mathrm{m})$ e ângulo inter-hastes idealizado para otimizar a força radial. A ligação interanel foi feita por meio de links curtos em formato sinusóide e espessura muito fina $(65$ $\mu \mathrm{m})$, com espaço circunferencial de $6 \mathrm{~mm}$ de diâmetro para acesso a ramo lateral. Dois outros animais receberam stents convencionais de controle para comparação (Driver ${ }^{\circledR}$, Medtronic Inc., e Matrix ${ }^{\circledR}$, Sahajanand Medical Technologies). Após um mês, os stents implantados foram retirados para análise microscópica. Resultados: Tanto o stent-teste como os stents comerciais de controle apresentaram hiperplasia neo-intimal discreta a moderada aos 30 dias, com obstrução neo-intimal de $30,0 \pm 12,2 \%$ e 22,4 $\pm 16,5 \%$, respectivamente. Todos os stents, no grupo stentteste e no grupo controle, encontravam-se com alto grau de endotelização, com discreto infiltrado inflamatório e persistência de fibrina discreta ou ausente. Conclusões: Esses achados sugerem que, do ponto de vista da resposta vascular ao implante, o novo stent apresentou desempe-

\section{SUMMARY}

\author{
Cobalt-Chromium Coronary Stent Conceived \\ in Brazil: Preliminary Histologic Findings in \\ an Experimental Porcine Model
}

Background: This study aims to evaluate, in an experimental porcine model, a new and pioneer cobalt-chromium thin-strut coronary stent conceived in Brazil. Also, it aims to report the initial experience of a Brazilian center for pre-clinical validation of endovascular devices. Method: The new bare Cobalt-Chromium stent (Scitech Produtos Médicos Ltda) was implanted in the coronary arteries of twelve domestic pigs. The stent was designed in short rings (for increased homogeneity of vessel scaffolding), thin struts $(75 \mu \mathrm{m})$ and inter-strut angle engineered to optimize the radial strength. The inter-ring connection was made by a short and very thin link $(65 \mu \mathrm{m})$ in a curved format, with a circumferential diameter of $6 \mathrm{~mm}$ for side branch access. Another two animals received conventional bare metal stents as controls (Driver $®$, Medtronic Inc., e Matrix $®$, Sahajanand Medical Technologies). After one month, the implanted stents were excised for microscopic analysis. Results: Both the test-stent and the control commercial stents had mild to moderate neointimal hyperplasia at 30 days, with a neointimal percent obstruction of $30.0 \pm 12.2 \%$ and $22.4 \pm 16.5 \%$, respectively. All stents, in the test-stent and the control groups, had a high degree of endothelialization with a mild inflammatory reaction and absent or mild persistence of fibrin. Conclusions: The present findings suggest

\footnotetext{
1 Instituto do Coração (InCor), Hospital das Clínicas da Faculdade de Medicina da Universidade de São Paulo, São Paulo, SP.

2 Instituto de Pesquisas Energéticas e Nucleares da Universidade de São Paulo e Innovatech Medical Ltda., São Paulo, SP.

3 Laboratório de Osteodistrofia Renal, LIM-16, Faculdade de Medicina da Universidade de São Paulo, São Paulo, SP.

Correspondência: Pedro A. Lemos. Hemodinâmica. Instituto do Coração (InCor) - HC-FMUSP. Av. Dr. Enéas Carvalho de Aguiar, 44 - Bloco I - 3o andar - São Paulo, SP - CEP 05403-000.

E-mail: pedro.lemos@incor.usp.br

Recebido em: 4/10/2007 • Aceito em: 26/11/2007
} 

Experimental Porcino. Rev Bras Cardiol Invas 2007; 15(4): 378-385.

nho semelhante ao de outros stents não-farmacológicos atualmente em uso comercial.

DESCRITORES: Angioplastia transluminal percutânea coronária. Próteses e implantes. Contenedores. that, as regards to the vascular reaction to the implant, the new stent presents a similar performance compared to other bare metal stents currently available for commercial use.

DESCRIPTORS: Percutaneous coronary transluminal angioplasty. Prostheses and implants. Stents.
0 implante de stent coronário foi rapidamente incorporado à prática clínica e hoje perfaz mais de $90 \%$ de todos os procedimentos percutâneos realizados em todo o mundo. Diversas melhorias técnicas têm sido progressivamente incorporadas aos stents, o que determinou a disponibilização atual de uma família de dispositivos com amplas possibilidades de aplicação em diversas situações clínicas e anatômicas.

Múltiplas estruturas de stents têm sido testadas nos últimos anos, incluindo stents tubulares ou coils, expansíveis por balão ou auto-expansíveis. Além disso, diferentes desenhos de malhas metálicas têm sido testados para aplicação em stents coronários. Curiosamente, os stents tubulares de aço inoxidável 316L confeccionados por corte a laser foram introduzidos já na primeira geração de stents e persistem, ainda hoje, como o tipo de stent mais utilizado. No entanto, mais recentemente, stents confeccionados a partir de ligas cobalto-cromo têm sido desenvolvidos com o intuito de melhorar algumas características dos stents tradicionais de aço inoxidável. Ligas metálicas de cobaltocromo apresentam maior resistência mecânica que o aço inoxidável, o que, em teoria, possibilita a confecção de stents com hastes mais finas sem que haja perda da força radial ou aumento do recoil do stent. Por sua vez, a redução da espessura das hastes possibilitaria a obtenção de stents mais flexíveis e com menor perfil de cruzamento. Evidências clínicas têm sugerido também que pacientes tratados com stents com hastes metálicas finas apresentam menor proliferação neo-intimal e melhor evolução que pacientes tratados com outros stents ${ }^{1,2}$.

Idealmente, novos dispositivos endovasculares devem ser exaustivamente testados em ambiente experimental antes de serem aplicados na prática clínica. Especificamente, stents coronários são freqüentemente testados em modelo porcino para avaliar a resposta biológica induzida pelo implante e analisar parâmetros objetivos de segurança e eficácia. Mesmo para stents coronários não-farmacológicos, cujo padrão de indução de reação vascular é relativamente bem documentado e conhecido, a fase de testes experimentais deve ser observada, especialmente quando da introdução de qualquer inovação tecnológica nos processos de confecção.

No presente estudo, são apresentados os achados preliminares dos testes experimentais de um novo stent tubular de corte a laser, de liga cobalto-cromo e de hastes finas, de pioneira concepção brasileira. Os resultados refletem a fase inicial de avaliação do novo stent em animais de experimentação, com ensaios também realizados de forma pioneira em centro de validação pré-clínica instalado no País.

\section{MÉTODO}

\section{Formato do estudo}

O estudo foi realizado em 12 porcos domésticos juvenis, não-ateroscleróticos, submetidos a implante de stent de liga cobalto-cromo e hastes finas nãofarmacológico (Scitech Produtos Médicos Ltda.) em uma das artérias coronárias (direita, descendente anterior ou circunflexa), escolhida de maneira aleatória. Nas outras artérias coronárias foram implantadas outras formulações de stents, cujos achados não serão relatados no presente estudo. Tal formato experimental se apóia no fato de que os stents locados em artérias não relacionadas tendem a apresentar comportamento e evolução independente, sendo tradicionalmente analisados de maneira isolada. Ainda, dois animais receberam stents convencionais de uso comercial $(n=2)$ para comparação (stent de liga cobalto-cromo Driver ${ }^{\circledR}$, Medtronic Inc., e stent de aço inoxidável Matrix ${ }^{\circledR}$, Sahajanand Medical Technologies). Após um mês do implante, os animais foram sacrificados e foram retirados os segmentos arteriais contendo os stents implantados para análise microscópica.

O presente estudo foi aprovado pelo Comitê de Ética Institucional e foi realizado em conformidade com as normas de Boas Práticas Laboratoriais.

\section{Procedimentos}

Todos os animais foram pré-tratados pelo menos um dia antes do procedimento com $75 \mathrm{mg}$ de clopidogrel e $100 \mathrm{mg}$ de aspirina, os quais foram mantidos por sete dias e até o fim do estudo, respectivamente. Os animais foram pré-anestesiados com bromazepan e a indução anestésica foi realizada com tiopental. Após a intubação orotraqueal, a anestesia foi mantida com isoflurano durante o procedimento.

O acesso vascular foi obtido por meio de dissecção cirúrgica da artéria femoral comum, com introdução de bainha vascular 6F sob visão direta. Após 
administração de 10.000 UI de heparina por via intravenosa, as artérias coronárias esquerda e direita foram canuladas seletivamente sob fluoroscopia, utilizandose cateteres-guia 6F. A aquisição de cinecoronariografia basal da artéria de interesse foi realizada em múltiplas projeções após injeção intracoronária de $200 \mu \mathrm{g}$ de nitroglicerina. Todos os procedimentos foram conduzidos em sala de hemodinâmica dedicada aos testes com animais de experimentação (Figura 1).

Sob fluoroscopia, uma corda-guia 0,014" foi manipulada até a porção distal da artéria-alvo, sobre a qual o stent foi dirigido até o segmento coronário de interesse. O diâmetro dos stents foi selecionado de forma a manter a relação stent/artéria final entre 1,1-1,2:1,0. Após o término do implante do stent, foram administrados $200 \mu \mathrm{g}$ de nitroglicerina intracoronária e foi realizada cinecoronariografia final (Figura 1).

Depois de um mês do implante, foi realizado novo estudo angiográfico (Figura 1), seguindo o mesmo protocolo descrito anteriormente para a angiografia índice (anestesia, acesso vascular e aquisição angiográfica). Ao término da angiografia de controle, o animal foi sacrificado após aprofundamento máximo da anestesia e overdose de cloreto de potássio.

\section{Características do novo stent de cobalto-cromo de hastes finas}

O stent em teste foi construído a partir de tubo metálico em liga de cobalto-cromo L605, cortado a laser e posteriormente submetido a tratamento térmico e decapagem química, e polido por eletroquímica seguida de passivação (Figura 2). O stent possuía um desenho em anéis corrugados, com espessura final das hastes de $75 \mu \mathrm{m}$ (Figura 3). Em cada anel, o ângulo final máximo entre as hastes era de 45 graus para o stent expandido, formato especificamente concebido com o intuito de otimizar a força radial final (Figura 2). Os anéis eram construídos em células curtas $(1,1 \mathrm{~mm})$, o que permite a ampliação do número de células e aumenta a homogeneidade da cobertura metálica em segmentos tortuosos e angulados (Figura 4). A ligação interanel foi feita por meio de links curtos em formato sinusóide (espessura de $65 \mu \mathrm{m}$ ), que proporcionavam espaço circunferencial máximo de aproximadamente $6 \mathrm{~mm}$ de diâmetro ("acesso a ramo lateral") (Figuras 2 e 5).

\section{Processamento e análise histológicos}

Imediatamente após o sacrifício, os corações foram explantados e perfundidos por 30 minutos sob pressão $(\sim 80 \mathrm{mmHg})$ através da raiz da aorta com solução de formalina a $10 \%$, sendo posteriormente fixados por imersão por 24 horas ou mais. Posteriormente, os segmentos arteriais de interesse e o tecido adjacente foram dissecados da superfície epicárdica para processamento histológico.
Os segmentos arteriais contendo os stents foram seccionados em três segmentos (proximal, médio e distal) e desidratados em soluções progressivas de etanol para posterior inclusão em resina plástica de metacrilato ${ }^{3}$. Após a inclusão em resina, foram obtidos cortes histológicos utilizando-se micrótomo adequado (Polycut S, Leica, Alemanha), equipado com lâmina de tungstênio específica (Figura 6). Para cada segmento foi obtido pelo menos um corte micrométrico com aproximadamente $5 \mu \mathrm{m}$ de espessura. Os cortes histológicos foram então desplastificados e corados para análise histológica com hematoxilina-eosina e coloração de Verhoeff-van Gieson. As características morfológicas dos cortes histológicos foram analisadas de acordo com os critérios descritos previamente:

1. Escore de endotelização do stent ${ }^{4,5}$ :

a. Grau 1: $<25 \%$ da circunferência da luz arterial recoberta por células endoteliais;

b. Grau 2: 25\%-75\% da circunferência da luz arterial recoberta por células endoteliais;

c. Grau 3: > 75\% da circunferência da luz arterial recoberta por células endoteliais.

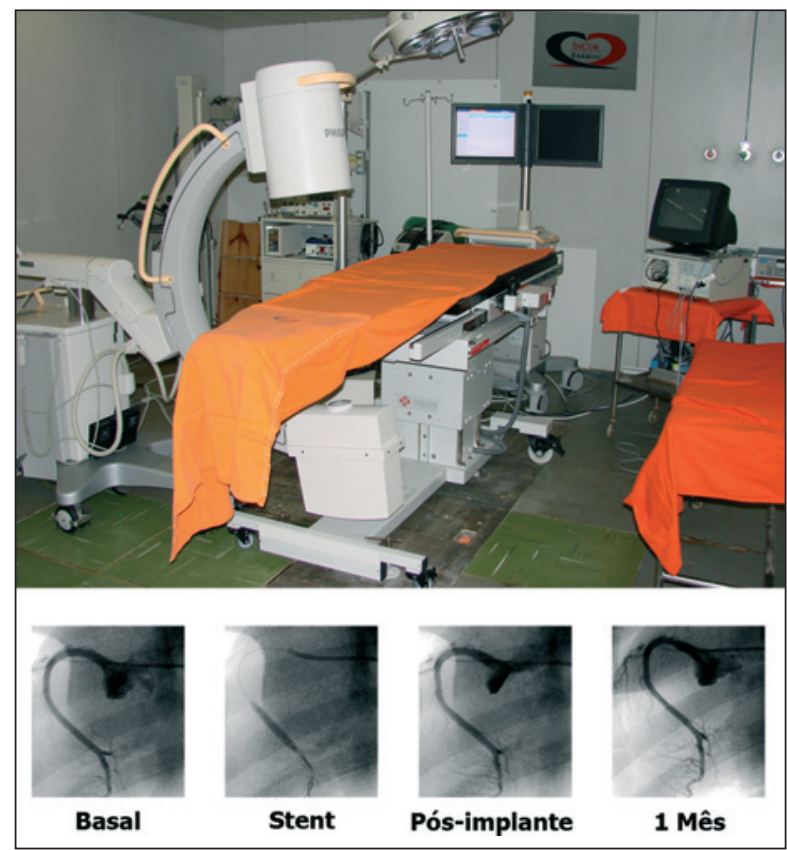

Figura 1 - Laboratório de Cardiologia Intervencionista Experimental, dedicado à cateterização coronária em animais de médio porte e com capacidade de realização de cineangiografia. Abaixo, é ilustrada a artéria coronária direita de um animal do estudo submetido a uma seqüência angiográfica nos tempos basal, implante do stent, pós-implante imediato e resultado tardio após um mês. Observe que o diâmetro do stent é selecionado para superestimar o tamanho da artéria em $10 \%$ a $20 \%$. No tempo tardio, nota-se discreta redução luminal, em comparação ao achado imediatamente após o implante, em decorrência da presença de discreta hiperplasia neo-intimal. 


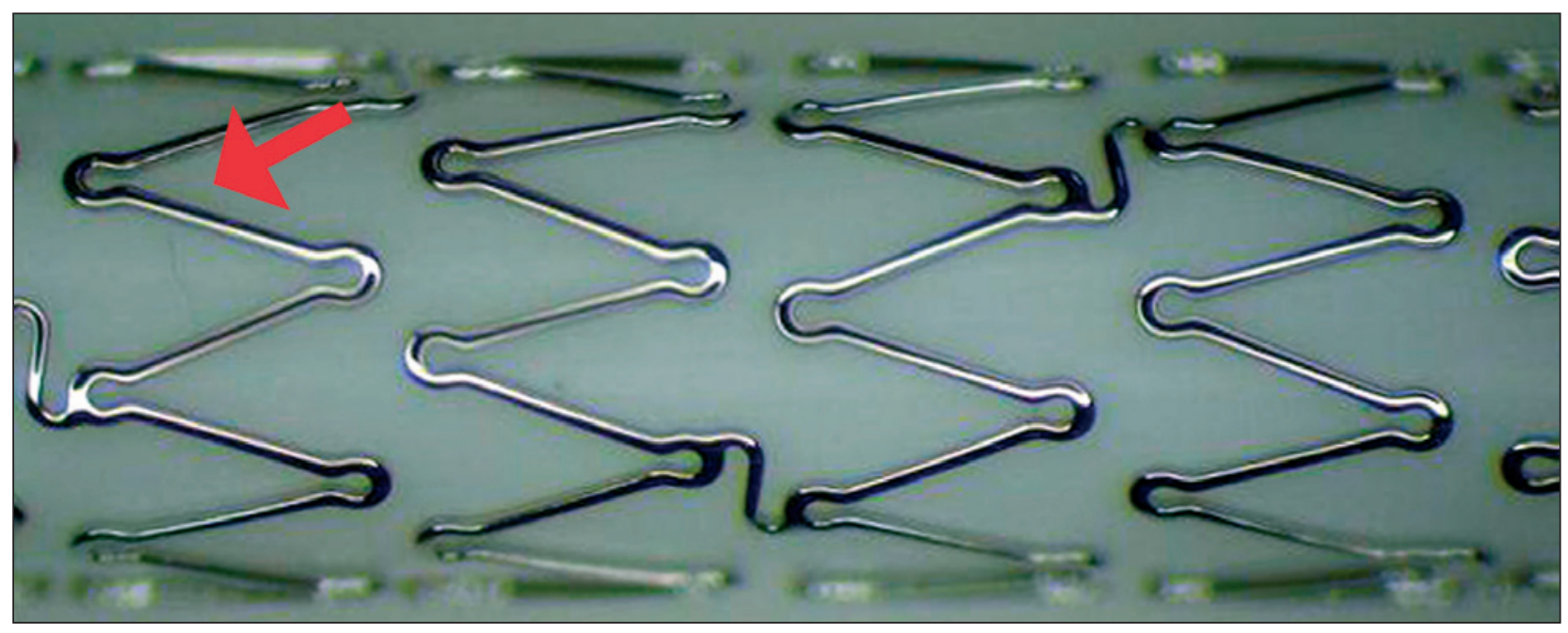

Figura 2 - Fotografia em grande aumento do stent metálico de liga de cobalto-cromo L605, após os polimentos elétrico e químico. A seta indica a angulação inter-hastes inferior a 45 graus (45 graus somente atingido como ângulo final máximo do stent expandido a 4,5 mm de diâmetro).

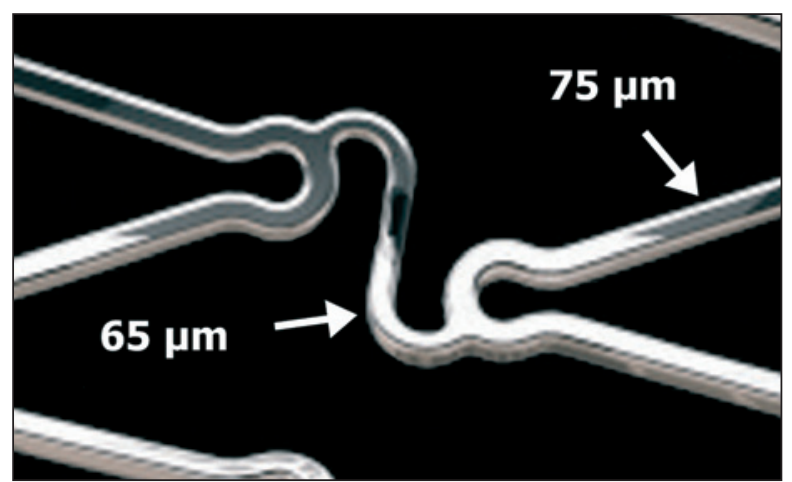

Figura 3 - Detalhe das hastes do stent (espessura de $75 \mu \mathrm{m}$ ) e do link interanel em formato sinusóide (espessura de $65 \mu \mathrm{m}$ ).

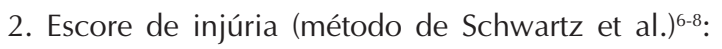

a. Grau 0: Lâmina elástica interna intacta; endotélio tipicamente desnudado; média comprimida, porém não lacerada;

b. Grau 1: Lâmina elástica interna lacerada; média tipicamente comprimida, mas não lacerada;

c. Grau 2: Lâmina elástica interna lacerada; média visivelmente lacerada; lâmina elástica externa intacta, porém comprimida;

d. Grau 3: Lâmina elástica externa lacerada; tipicamente grandes lacerações da média se estendendo até a lâmina elástica externa; hastes do stent por vezes locadas na adventícia.

3. Escore de injúria (método de Gunn et al.) ${ }^{9}$ :

a. Grau 0: Sem deformação;

b. Grau 1: Lâmina elástica interna deformada $<45$ graus; c. Grau 2: Lâmina elástica interna deformada $>45$ graus;

d. Grau 3: Lâmina elástica interna rota;

e. Grau 4: Lâmina elástica externa rota.

4. Escore de inflamação $0^{4,5,7,10}$ :

a. Grau 0: Ausente;

b. Grau 1: Células inflamatórias esparsas;

c. Grau 2: Células inflamatórias ao redor de 50\% da haste do stent em pelo menos $25 \%$ a $50 \%$ da circunferência da artéria;

d. Grau 3: Células inflamatórias envolvendo totalmente a haste do stent em pelo menos $25 \%$ a $50 \%$ da circunferência da artéria.

5. Conteúdo de fibrina da íntima ${ }^{4,5}$ :

a. Grau 0: Ausente;

b. Grau 1: Focos de fibrina residual envolvendo qualquer porção da artéria OU deposição moderada de fibrina adjacente à haste envolvendo $<25 \%$ da circunferência da artéria;

c. Grau 2: Deposição moderada de fibrina envolvendo > 25\% da circunferência da artéria OU deposição acentuada envolvendo $<25 \%$ da circunferência da artéria;

d. Grau 3: Deposição acentuada envolvendo > $25 \%$ da circunferência da artéria.

Além disso, foi também anotada a descrição subjetiva de outros achados, incluindo afilamento da média, perda de celularidade, hialinização, reação de corpo estranho, áreas de necrose e trombose ${ }^{6}$.

Foram analisados os seguintes parâmetros histomorfométricos: área luminal, área delimitada pela lâmina 


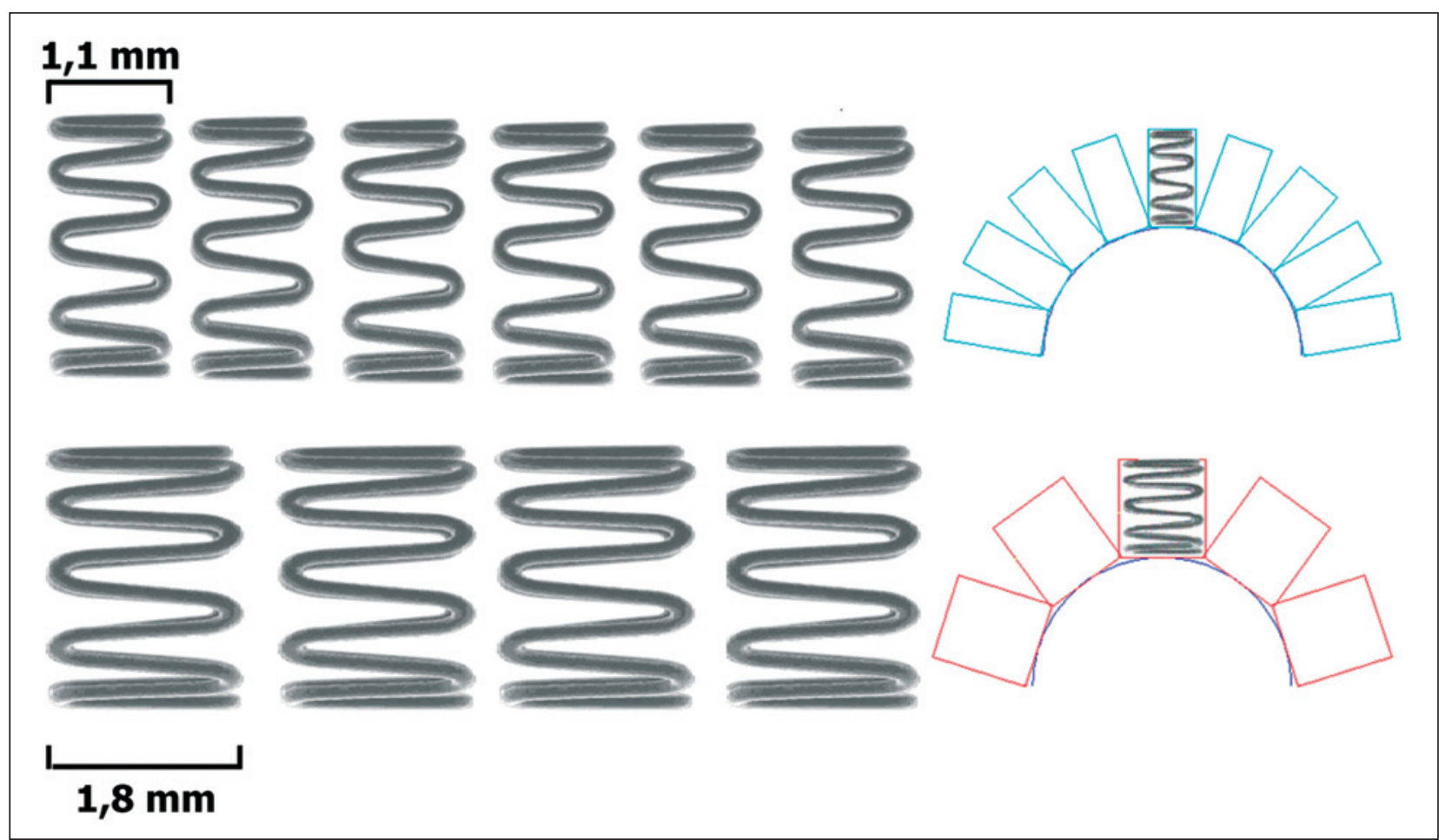

Figura 4 - Esquema comparativo do impacto da redução do comprimento dos anéis sobre a flexibilidade do stent e o efeito "escama" em segmentos tortuosos. Acima é ilustrado o stent em teste com comprimento de anel de 1,1 mm e abaixo, stent teórico com células mais longas $(1,8 \mathrm{~mm})$. Nos painéis à direita, observa-se menor espaçamento entre os anéis e cobertura mais homogênea do stent com anéis mais curtos quando aplicado sobre segmento angulado.

elástica interna, área delimitada pela lâmina elástica externa, área neo-intimal, área da média, área delimitada do stent, espessura neo-intimal medida no ponto médio entre cada par de hastes de stent e em cada haste de stent (distância haste-lúmen) $)^{6,11,12}$.

\section{Análise de dados}

Os achados histológicos e morfométricos são descritos por meio da média (variáveis contínuas) ou da mediana (variáveis ordinais, isto é, escores) de todos os cortes avaliados em uma mesma artéria. Para fins deste relato preliminar, os dados são apresentados de

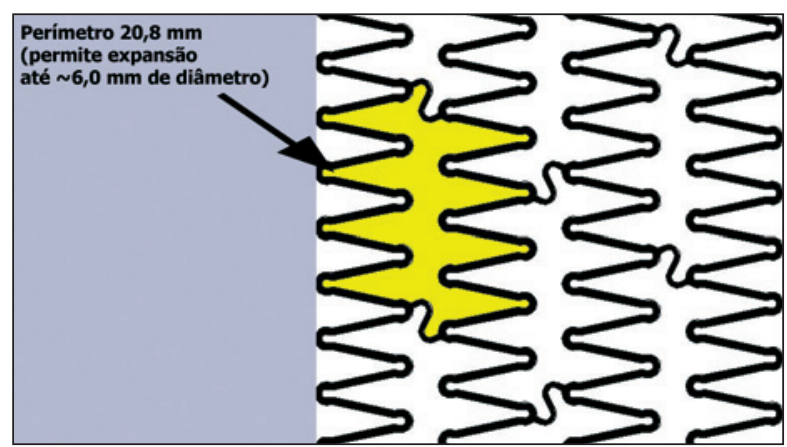

Figura 5 - Esquema ilustrativo do espaço circunferencial lateral do stent ("acesso ao ramo lateral"). maneira descritiva, sem análise estatística de inferência formal, em decorrência do tamanho da amostra.

\section{RESULTADOS}

Todos os stents foram implantados sem intercorrências ou dificuldades técnicas. No total, foram incluídos 14 porcos, que receberam implante de 12 stents-teste e 2 stents não-farmacológicos de uso comer-

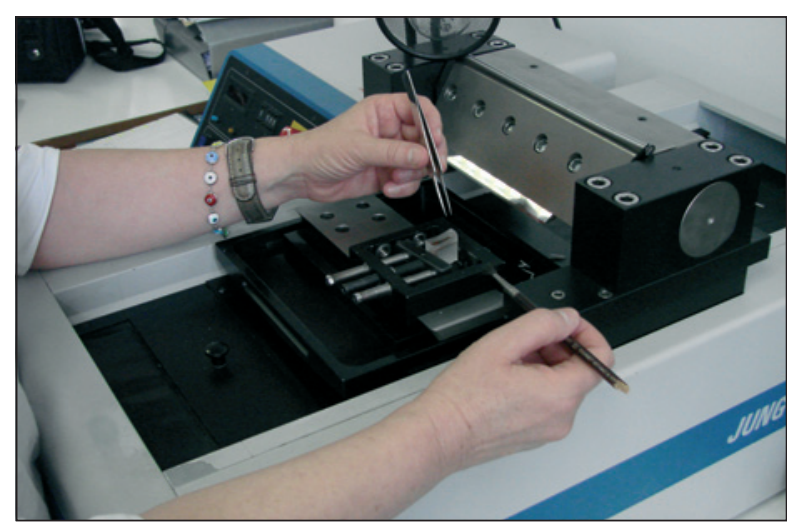

Figura 6 - Micrótomo com lâmina de tungstênio em atividade para obtenção de cortes histológicos de artéria com stent incluída em resina de metacrilato. Note o bloco de tecido em resina posicionado para corte. 
cial para controle. Após um mês, todos os stents encontravam-se pérvios. Foram obtidos 42 cortes histológicos seccionais para análise.

À análise histológica, observou-se que o implante do stent (relação balão:artéria 1,1-1,2:1,0) induziu injúria vascular de grau leve a moderado tanto nos stentsteste como nos stents comerciais de controle (Figura 7 e Tabela 1). Após um mês, todos os stents encontravam-se com alto grau de endotelização, com discreto infiltrado inflamatório e persistência de fibrina discreta ou ausente (Tabela 1 e Figura 8). Não foram identificadas reações de corpo estranho, necrose, malaposição das hastes ou outros achados morfológicos significativos. Os achados histomorfométricos, apresentados na Tabela 2, demonstram área do stent semeIhante à área da lâmina elástica interna em ambos os grupos. Tanto o stent-teste como os stents comerciais de controle apresentaram hiperplasia neo-intimal discreta a moderada, com obstrução neo-intimal de 30,0 $\pm 12,2 \%$ e $22,4 \pm 16,5 \%$, respectivamente.

\section{DISCUSSÃO}

Segundo os principais achados deste estudo preliminar, o novo stent-teste de cobalto-cromo com has- tes finas induziu a resposta vascular clássica, habitualmente observada em stents metálicos não-farmacológicos. Essa resposta foi comparável, em intensidade e características estruturais, à dos stents comerciais utilizados como controle, bem como à observada nos inúmeros estudos prévios da literatura com esse tipo de stent ${ }^{9,13}$. Depois de um mês do implante em modelo de hiperplasia neo-intimal em artérias coronárias de porcos, não houve surgimento de resposta hiperproliferativa ou de achados compatíveis com toxicidade local. O espessamento intimal observado após o implante do novo stent foi secundário ao surgimento de tecido neointimal de discreta a moderada importância, sem persistência tardia de fibrina, sem sinais de reação inflamatória importante e com reendotelização completa.

O novo stent testado foi idealizado para atender a um conjunto de características a priori consideradas necessárias a um stent coronário ideal. A estrutura de hastes finas $(<100 \mu \mathrm{m}$ de espessura) tem sido associada a menor índice de reestenose em pacientes ${ }^{1,2}$, ao mesmo tempo que possibilita a confecção de um stent com baixo perfil de cruzamento. Em especial, a conformação da malha do novo stent foi desenhada de maneira a permitir bom desempenho em curvaturas, pela engenharia em anéis curtos $(1,1 \mathrm{~mm}$ de

TABELA 1

Achados morfológicos à análise histológica após um mês

\begin{tabular}{|c|c|c|}
\hline & Stent-teste & Stent comercial de controle* \\
\hline Injúria vascular (escore de Schwartz) & $1,5[1-2]$ & $1,5[1-2]$ \\
\hline Injúria vascular (escore de Gunn9) & $2,5[2-3]$ & $2[2-2]$ \\
\hline Endotelização $\mathrm{O}^{4,5}$ & $3[3-3]$ & $3[3-3]$ \\
\hline Inflamação ${ }^{4,5,10}$ & $1[1-1]$ & $1,5[1-2]$ \\
\hline Conteúdo de fibrina da íntima ${ }^{4,5}$ & $0[0-1]$ & $0,5[0-1]$ \\
\hline
\end{tabular}

Números são mediana [intervalo interquartil]

TABELA 2

Achados histomorfométricos após um mês

\begin{tabular}{lcc}
\hline & Stent-teste & Stent comercial de controle* \\
\hline Área luminal $\left(\mathrm{mm}^{2}\right)$ & $2,28 \pm 0,81$ & $3,77 \pm 0,50$ \\
Área da LEI $\left(\mathrm{mm}^{2}\right)$ & $3,51 \pm 1,01$ & $5,03 \pm 1,72$ \\
Área da LEE $\left(\mathrm{mm}^{2}\right)$ & $4,49 \pm 1,17$ & $6,27 \pm 0,29$ \\
Área do stent $\left(\mathrm{mm}^{2}\right)$ & $3,43 \pm 0,83$ & $5,27 \pm 2,41$ \\
Área de neo-íntima $\left(\mathrm{mm}^{2}\right)$ & $1,03 \pm 0,46$ & $1,26 \pm 1,21$ \\
Espessura da neo-íntima inter-hastes $(\mathrm{mm})$ & $0,17 \pm 0,09$ & $0,13 \pm 0,14$ \\
Espessura da neo-íntima sobre a haste $(\mathrm{mm})$ & $0,17 \pm 0,09$ & $0,20 \pm 0,26$ \\
Obstrução neo-intimal $(\%)$ & $30,0 \pm 12,2$ & $22,4 \pm 16,5$ \\
Área da média $\left(\mathrm{mm}^{2}\right)$ & $0,89 \pm 0,37$ & $1,25 \pm 0,57$ \\
\hline
\end{tabular}

LEE = lâmina elástica externa; LEI = lâmina elástica interna.

Números são média (desvio padrão). 
Lemos PA, et al. Stent Coronário de Liga Cobalto-Cromo Concebido no Brasil: Achados Histológicos Preliminares em Modelo Experimental Porcino. Rev Bras Cardiol Invas 2007; 15(4): 378-385.

comprimento) ligados por hastes curvas extremamente finas $(65 \mu \mathrm{m})$. A distribuição e a angulação das hastes foram concebidas de maneira a permitir amplo acesso a ramos laterais sem prejuízo da força radial do stent. Dessa forma, os achados histológicos são promissores, pois apontam para um stent com concepção mecânica avançada, sem comprometer as características de reação vascular biológica esperada para um stent não-farmacológico.

As atividades de pesquisa, desenvolvimento e inovação no campo da intervenção percutânea coronária têm sido alvo de intenso interesse em diversos países do mundo. São inúmeros e complexos os processos a serem implementados para culminar com um produto final, testado, para uso clínico e passível de ser produzido e distribuído em escala comercial. Essa cadeia de eventos, que se inicia com a concepção de um novo dispositivo endovascular, prossegue com as etapas de confecção de protótipos, testes de validação pré-clínicos (mecânicos e biológicos in vitro e in vivo) e, por fim, testes de segurança e eficácia em humanos (estes últimos habitualmente conduzidos em quatro fases sucessivas). É importante ressaltar que, apesar do amplo parque industrial instalado em vários países, o desenvolvimento pioneiro não conta com a possibilidade de transferência de tecnologia, quase sempre mantida como segredo industrial e não disponível para consulta pública.

É nesse contexto que se insere a experiência inicial apresentada neste trabalho. A concepção e a produção de stents metálicos com corte a laser em um cenário pioneiro não prescinde de intenso trabalho de prospecção e análise das características mecânicas e físicas dos materiais utilizados, do design da malha metálica, dos processos de corte a laser e dos detalhes

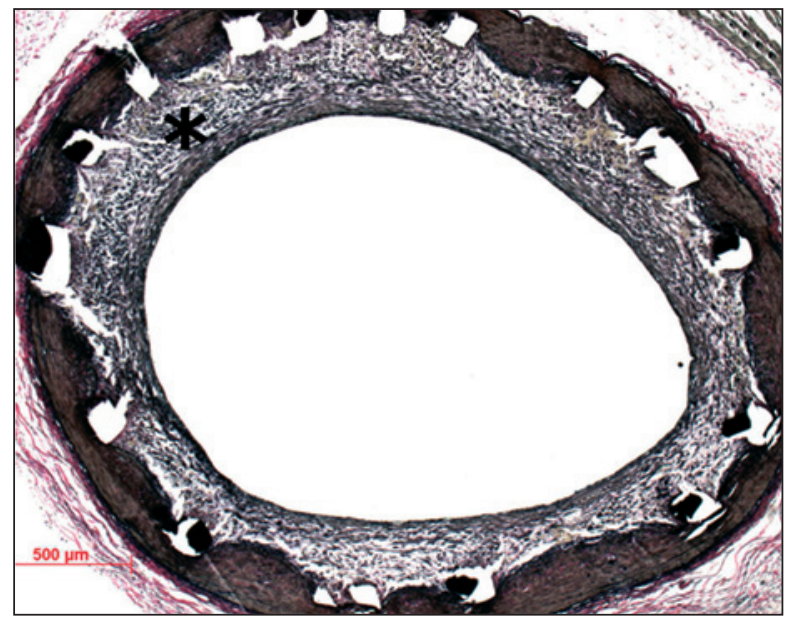

Figura 7 - Fotomicrografia óptica de artéria coronária com stentteste de cobalto-cromo um mês após implante em modelo porcino. Observa-se espessamento neo-intimal (asterisco), secundário à injúria mecânica discreta/moderada pelo implante do stent (escore de Schwartz $^{6}$ grau I e escore de Gunn ${ }^{9}$ grau II). dos tratamentos térmico, químico e eletroquímico do dispositivo. A ausência de atividade fabril ou de pesquisa e desenvolvimento básicos nesse ramo de atividade, no Brasil, determina carência de instituições acadêmicas ou não-acadêmicas dedicadas à realização de testes de validação pré-clínicos. Os resultados descritos neste estudo traduzem o início das atividades de um Laboratório de Cardiologia Intervencionista Experimental, também pioneiro no País, especificamente organizado e equipado para a avaliação científica de dispositivos e procedimentos de intervenção percutânea, com capacidade instalada para cineangiografia e implante de stents em artérias coronárias de animais de médio porte. É importante salientar que foram também desenvolvidas as habilidades para o processamento histológico de vasos com implante de stent sem necessidade de retirada do material metálico para obtenção dos cortes histológicos, passo que

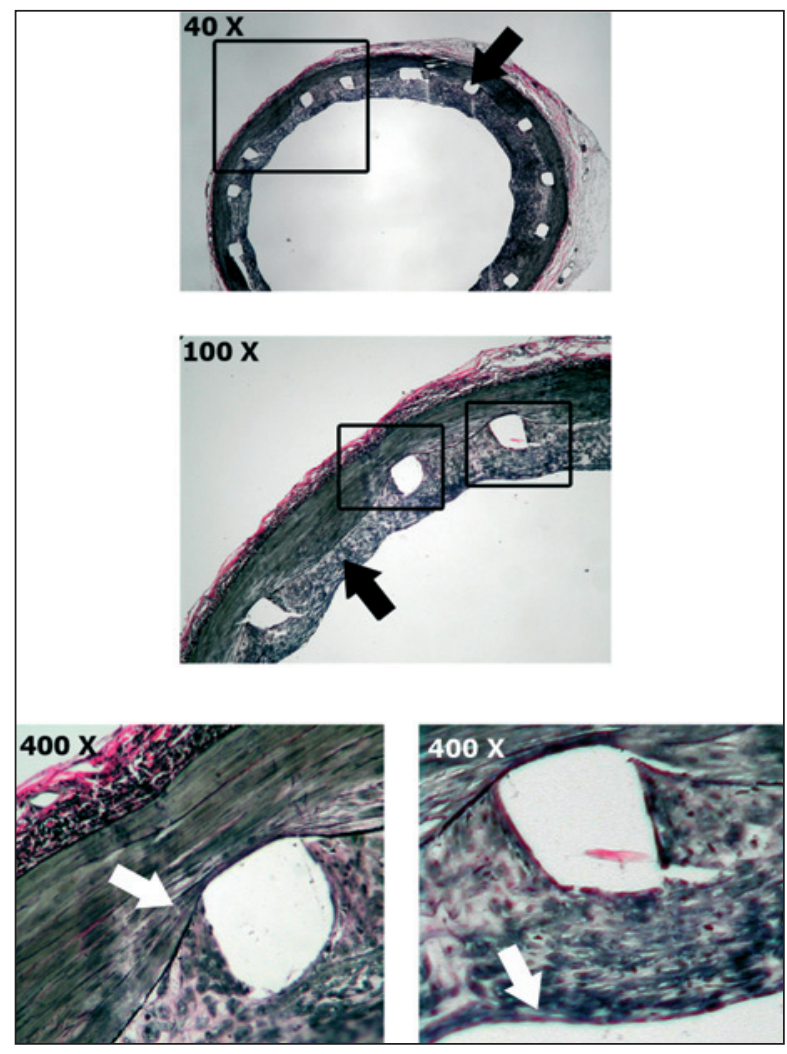

Figura 8 - Corte transversal de artéria coronária com stent-teste de cobalto-cromo um mês após o implante em modelo porcino. Os locais em branco, de formato aproximadamente quadrado, correspondem às secções das hastes do stent (painel superior, seta escura indicando uma das hastes). Observa-se nos painéis superior e médio a presença de neo-íntima homogênea (seta escura no painel médio), sem fibrina persistente e sem sinais de trombo local. A camada média, corada em cinza escuro relativamente homogêneo, está comprimida nos pontos com stent, como se pode perceber no painel médio, realçado em detalhe no painel inferior (seta branca, à esquerda). Nota-se ainda a presença de recobrimento endotelial íntegro (seta branca, painel inferior à direita). Coloração pelo Verhoeff-van Gieson; aumentos de 40X, 100X e 400X. 
freqüentemente configura um dos pontos mais limitantes no processo. A inclusão em resina de metacrilato e o corte micrométrico de espécime contendo metal (sem que haja danificação dos tecidos adjacentes às hastes do stent) constituem as principais dificuldades que restringem o processamento histológico de stents a poucos centros no mundo. Os resultados apresentados traduzem o aprimoramento da técnica histológica em nosso laboratório, atualmente com bom aproveitamento e qualidade dos cortes para análise histológica final, como ilustrado nas figuras apresentadas.

\section{CONCLUSÃO}

Em conclusão, os resultados preliminares após o implante experimental de novo stent de liga cobaltocromo são compatíveis com o padrão esperado para um stent coronário não-farmacológico. Esses achados sugerem que, do ponto de vista da resposta vascular ao implante, o novo stent tem desempenho semelhante ao de outros stents não-farmacológicos atualmente em uso comercial.

\section{AGRADECIMENTOS}

Os autores agradecem à sra. Leonora Loppnow, pela dedicação e empenho no preparo e execução deste estudo, e às sras. Andréa R. de Carvalho e Fernanda Giordano, pelo trabalho de captação de recursos e gestão de projetos em ciência, tecnologia e inovação.

\section{CRÉDITOS}

O presente estudo faz parte do Programa de Desenvolvimento Nacional de Endopróteses Vasculares (stents) - PDNS, iniciado em 2004-2005. Conta com o suporte da Secretaria de Ciência, Tecnologia e Insumos Estratégicos (SCTIE)/Departamento de Ciência e Tecnologia (DECIT) do Ministério da Saúde (MS), do Conselho Nacional de Desenvolvimento Científico e Tecnológico ( $\mathrm{CNPq}$ ) e da Financiadora de Estudos e Projetos (FINEP) do Ministério da Ciência e Tecnologia (MCT). O desenvolvimento do processo de corte a laser de stents contou com o apoio da Fundação de Amparo à Pesquisa do Estado de São Paulo (FAPESP; Forma de Apoio: Inovação Tecnológica - Pesquisa Inovativa na Pequena e Micro Empresa [PIPE]).

\section{NOTAS}

O dr. Spero P. Morato é diretor-presidente da Innovatech Medical Ltda. Os drs. Pedro A. Lemos, Expedito E. Ribeiro e Francisco R. M. Laurindo são consultores científicos da Scitech Prod. Médicos. Os drs. Pedro A. Lemos e Expedito E. Ribeiro receberam honorários eventuais em palestras nacionais e internacionais pela Boston Scientific e Cordis. O dr. Expedito E. Ribeiro recebeu honorários eventuais em palestras nacionais e internacionais pela Merck Sharp \& Dohme e Sanofi-Aventis. O dr. Pedro A. Lemos é integrante do Boston Scientific Latin America SCIMAB (Scientific Medical Advisory Board). O dr. Expedito E. Ribeiro é integrante do Advisory Board for New Products and Developments, da Cordis. O dr. Eulógio E. Martinez é integrante do Advisory Board para Heparinas de Baixo Peso Molecular da Sanofi-Aventis.

\section{REFERÊNCIAS BIBLIOGRÁFICAS}

1. Kastrati A, Mehilli J, Dirschinger J, Dotzer F, Schuhlen H, Neumann FJ, et al. Intracoronary stenting and angiographic results: strut thickness effect on restenosis outcome (ISARSTEREO) trial. Circulation. 2001;103(23):2816-21.

2. Pache J, Kastrati A, Mehilli J, Schuhlen H, Dotzer F, Hausleiter $J$, et al. Intracoronary stenting and angiographic results: strut thickness effect on restenosis outcome (ISAR-STEREO2) trial. J Am Coll Cardiol. 2003;41(8):1283-8.

3. van Beusekom HMM, Whelan DM, van de Plas $M$, van der Giessen W. A practical and rapid method of histological processing for examination of coronary arteries containing metallic stents. Cardiovasc Pathol. 1996;5(2):69-76.

4. Suzuki T, Kopia G, Hayashi S, Bailey LR, Llanos G, Wilensky $\mathrm{R}$, et al. Stent-based delivery of sirolimus reduces neointimal formation in a porcine coronary model. Circulation. 2001;104(10):1188-93.

5. Carter AJ, Aggarwal M, Kopia GA, Tio F, Tsao PS, Kolata $\mathrm{R}$, et al. Long-term effects of polymer-based, slow-release, sirolimus-eluting stents in a porcine coronary model. Cardiovasc Res. 2004;63(4):617-24.

6. Schwartz RS, Edelman ER, Carter A, Chronos N, Rogers C, Robinson KA, et al. Drug-eluting stents in preclinical studies: recommended evaluation from a consensus group. Circulation. 2002;106(14):1867-73.

7. Lowe HC, Schwartz RS, Mac Neill BD, Jang IK, Hayase M, Rogers C, et al. The porcine coronary model of in-stent restenosis: current status in the era of drug-eluting stents. Catheter Cardiovasc Interv. 2003;60(4):515-23.

8. Schwartz RS, Chronos NA, Virmani R. Preclinical restenosis models and drug-eluting stents: still important, still much to learn. J Am Coll Cardiol. 2004;44(7):1373-85.

9. Gunn J, Arnold N, Chan KH, Shepherd L, Cumberland DC, Crossman DC. Coronary artery stretch versus deep injury in the development of in-stent neointima. Heart. 2002;88(4):401-5.

10. Kornowski R, Hong MK, Tio FO, Bramwell O, Wu H, Leon $\mathrm{MB}$. In-stent restenosis: contributions of inflammatory responses and arterial injury to neointimal hyperplasia. J Am Coll Cardiol. 1998;31(1):224-30.

11. Heldman AW, Cheng L, Jenkins GM, Heller PF, Kim DW, Ware Jr. M, et al. Paclitaxel stent coating inhibits neointimal hyperplasia at 4 weeks in a porcine model of coronary restenosis. Circulation. 2001;103(18):2289-95.

12. Ahn YK, Jeong MH, Kim JW, Kim SH, Cho JH, Cho JG, et al. Preventive effects of the heparin-coated stent on restenosis in the porcine model. Catheter Cardiovasc Interv. 1999;48(3):324-30.

13. Yorozuya $M$, Suzuki $H$, Iso $Y$, Shibata $M$, Nakatani $M$, Koba $S$, et al. Comparison of the morphological changes of restenosis after the implantation of various types of stents in a swine model. Coron Artery Dis. 2002;13(6):305-12. 